\title{
IUCN Red List and the value of integrating genetics
}

\author{
Brittany A. Garner ${ }^{1}$ (1) . Sean Hoban ${ }^{2}$. Gordon Luikart ${ }^{3}$
}

Received: 2 January 2020 / Accepted: 4 August 2020 / Published online: 18 September 2020

(c) The Author(s) 2020

\begin{abstract}
Many species on endangered species lists such as the IUCN Red List (RL) are categorized using demographic factors such as numbers of mature individuals. Genetic factors are not currently used in the RL even though their explicit consideration, including effective population size $\left(N_{\mathrm{e}}\right)$ and expected heterozygosity-loss $(H$-loss), could improve the assessment of extinction risk. Here, we consider the estimation of $N_{\mathrm{e}}$ and $H$-loss in the context of RL species. First, we investigate the reporting of number of mature individuals for RL Endangered species, which is needed to estimate $N_{\mathrm{e}}$ and $H$-loss. We found 77\% of species assessments studied here did not report methods used to estimate the number of mature adults, and that these assessments rarely report other important determinants of $N_{\mathrm{e}}$ (e.g., sex ratio, variance in family size). We therefore applied common rules of thumb to estimate $N_{\mathrm{e}}$, and found that $N_{\mathrm{e}}$ was likely $<50$ for at least $25 \%$ of the 170 RL Endangered species studied here. We also estimated mean expected $H$-loss for these species over the next 100 years, and found it to be $9-29 \%$. These estimates of high $H$-loss and low $N_{\mathrm{e}}$ suggest that some species listed as Endangered likely warrant listing as Critically Endangered if genetic considerations were included. We recommend that RL and other assessment frameworks (i) report methods used for estimating the number of mature adults, (ii) include standardized information on species traits that influence $N_{\mathrm{e}}$ to facilitate $N_{\mathrm{e}}$ estimation, and (iii) consider using concepts like $N_{\mathrm{e}}$ and heterozygosity-loss in risk assessments.
\end{abstract}

Keywords Biodiversity preservation - Conservation genetics $\cdot$ Extinction risk · Effective size $\cdot$ Number of breeders · Population bottleneck

\section{Introduction}

Electronic supplementary material The online version of this article (https://doi.org/10.1007/s10592-020-01301-6) contains supplementary material, which is available to authorized users.

Brittany A. Garner

brittany.garner@umontana.edu

Sean Hoban

shoban@mortonarb.org

Gordon Luikart

gordon.luikart@mso.umt.edu

1 Interdisciplinary Degree Program and W.A. Franke College of Forestry \& Conservation, University of Montana, 32 Campus Dr, Missoula, MT 59812, USA

2 Center for Tree Science, The Morton Arboretum, 4100 Illinois Rt 53, Lisle, IL 60532, USA

3 Flathead Lake Biological Station, Division of Biological Sciences, University of Montana, 32125 Bio Station Ln, Polson, MT 59860, USA
The one process now going on that will take millions of years to correct is the loss of genetic and species diversity by the destruction of natural habitats. This is the folly our descendants are least likely to forgive us. -Edward O. Wilson, 1984

Biodiversity loss is among the most urgent problems facing the world today. The most recognized worldwide index for biodiversity is the International Union for Conservation of Nature's (IUCN) Red List. This list results from a large, informative, and continually updated database dedicated to "providing the world with the most objective, scientifically-based information on the current status of globally threatened biodiversity" (IUCN 2001). For extant organisms with adequate demographic data, the IUCN Red List assigns an extinction risk category ("Least Concern", "Near Threatened", "Vulnerable", "Endangered", or "Critically Endangered") based upon a variety of criteria (Mace and Lande 1991; IUCN 2001). The IUCN bases most of 
its risk assessment on factors regarding number of mature individuals, trend, and geographic range. The IUCN recognizes genetic diversity as one aspect of species diversity and health (Norse et al. 1986, Reed and Frankham 2003); however, genetic factors are seldom used explicitly in RL assessments (Laikre et al. 2009), or in conservation policy or assessments in general (Pierson et al. 2016). For example, Laikre (2010) concluded that genetic diversity was not monitored, genetic change indicators were missing, and no strategy had emerged for including genetic aspects into global biodiversity targets, a point reiterated in Laikre et al. 2020.

Genetic principles and parameters have been useful in assessing conservation priority and risk assessment in a range of taxa, particularly when extinction risks are difficult to evaluate from ecological and demographic data alone (Dunham et al. 1999). Effective population size $\left(N_{\mathrm{e}}\right)$ is defined as the size of the ideal population with the same rate of genetic drift as in the actual population being considered (Fisher 1930; Wright 1931). $N_{\mathrm{e}}$ is among the most important genetic parameters in evolutionary and conservation biology because it influences the rate of inbreeding, loss of genetic diversity, efficiency of natural selection, and the maintenance of evolutionary potential (Newman and Pilson 1997; Waples et al. 2014; Beaumont and Wang 2019). This is in contrast to the population census size, $N_{\mathrm{c}}$, which is often defined as the number of mature (adult) individuals (e.g., Frankham1995; Waples 2005; Waples et al. 2014). $N_{\mathrm{e}}$ (and the $N_{\mathrm{e}} / N_{\mathrm{c}}$ ratio) is often particularly small for species with high fecundity, high mortality in early life stages (type III survivorship), high sex ratio skew, polygamy, and/or heritability of reproductive success (Waples et al. 2014; Kendall et al. 2016; Wang 2016; Greenbaum et al. 2017; Sun and Hedgecock 2017).

However, the main driver of low $N_{\mathrm{e}}$ and $N_{\mathrm{e}} / N_{\mathrm{c}}$ ratios is typically high variation in reproductive success among individuals, which could be due to body size and fecundity variation (e.g., large trees or fish producing thousands of seeds or eggs), behavior (e.g., dominant males; Beletsky and Orians 1989), or chance. While some of the extremely low $N_{\mathrm{e}} / N_{\mathrm{c}}$ ratios reported in the literature have been contested as potential artifacts of sampling (Hauser et al. 2002; Ficetola et al. 2010; Waples 2016), the ratios in many species are often small $(<0.10)$. $N_{\mathrm{e}}$ is often small $(<50)$ and/or declining which is problematic for population persistence, and thus is of concern to conservation biologists (Allendorf and Ryman 2002, Laikre et al. 2020).

$N_{\mathrm{e}}$ ranging from around 50 to several hundred is within the range where genetic variation is lost rapidly due to genetic drift and deleterious effects of inbreeding likely occur; $N_{\mathrm{e}}$ below 50 signals critical and rapid genetic erosion (Frankham et al. 2002; Hoarau et al. 2005). This is especially true if the population size has been small for multiple generations and was recently large, because large (outbred) populations carry a large genetic load (deleterious alleles; Allendorf et al. 2013; Spigler et al. 2017). At small and declining $N_{\mathrm{e}}$, loss of allelic diversity is especially rapid and increases susceptibility to infectious disease and cancers (Ujvari et al. 2018). Thus, $N_{\mathrm{e}}$ could inform managers and other conservation stakeholders about a population's ability to persist and respond to environmental change, which is of great importance in the Anthropocene.

While there are increasingly useful genetic methods to estimate $N_{\mathrm{e}}$ (e.g., those based on linkage disequilibrium or sibship; Waples and Do 2008; Wang et al. 2016; Beaumont and Wang 2019) and genetic data are increasingly affordable, $N_{\mathrm{e}}$ need not be empirically measured with molecular markers for the $N_{\mathrm{e}}$ concept to be useful in assessing risk of a species or population. For example, the $N_{\mathrm{e}}$ for a species or taxonomic group is known to often be only $10 \%$ to $20 \%$ of the $N_{\mathrm{c}}$ (Frankham et al. 2014), and sometimes far less (e.g., $<1 \%$; Palstra and Ruzzante 2008). Biologists can estimate or approximate $N_{\mathrm{e}}$ from $N_{\mathrm{c}}$ using only demographic data such as the number of reproducing males and females, the adult sex ratio, longevity, family size variance, and more (Waples et al. 2013), if such information is available. This would allow explicit consideration that if $N_{\mathrm{e}} \ll 50$ (e.g., 20 to 30 ), then inbreeding depression (and fixation of deleterious alleles) and loss of alleles is likely to threaten a population's growth and persistence (Bozzuto et al. 2019). Furthermore, $N_{\mathrm{e}}$ estimates allow estimation of the loss of heterozygosity ( $H$-loss) expected over 100 years, for example, if the generation interval is known or approximated. Heterozygosity loss over 100 years (e.g., $>5 \%$ or $10 \%$ ) has been proposed as threshold for population extinction risk and management concern (e.g., Allendorf and Ryman 2002).

Our overarching goal here is to consider the use of $N_{\mathrm{e}}$ concepts and estimates, and loss of heterozygosity for IUCN Red List assessment procedures. Our main objectives are to (1) assess the standardization of reporting the "number of mature individuals" as estimated and reported within the IUCN Red List to facilitate $N_{\mathrm{e}}$ estimation, (2) estimate the $N_{\mathrm{e}}$ for species listed as endangered (EN) on the IUCN Red List per Criterion D using a range of generally accepted and reasonable $N_{\mathrm{e}} / N_{\mathrm{c}}$ ratios, (3) estimate the heterozygosity expected to be lost in the next 100 years based on those $N_{\mathrm{e}}$ estimations and generation interval estimates, and (4) identify which species listed as EN are at the most risk and could warrant listing as critically endangered (CR) if the $N_{\mathrm{e}}$ and heterozygosity-loss are considered. We predict that many species in the IUCN Red List are likely to have a small $N_{\mathrm{e}} / N_{\mathrm{c}}$ ratio and $N_{\mathrm{e}}<50$ (for multiple generations) and thus could benefit from revision of Red List ranking along with monitoring or management actions to prevent excessive loss of genetic variation and reduced probability of persistence (Crow and Kimura 1970; Allendorf and Ryman 2002; Lacy 2019). 


\section{Methods}

To assess and quantify standardization in the IUCN Red List reporting of number of mature individuals in a species, we first compared the text of recent available Red List guidelines, beginning from ones published in 2004 up to the most recent one, Version 14 (IUCN 2019). Similarities and differences between the guideline versions over time were recorded. We were interested in species listed as Endangered (EN) on the IUCN Red List as they are already of high conservation concern and are one risk category away from being Critically Endangered (CR), which is the most endangered category (except Extinct in the Wild). Under Criterion D, species with number of mature individuals $<250$ are listed as $\mathrm{EN}$, and $<50$ are listed as CR.

To analyze the assessments of EN species listed under Criterion D, we filtered all species assessments currently available on the IUCN Red List $(n=105,732)$ to include only those species listed as Endangered (EN; $n=9754)$, and then filtered to include only those categorized as EN under Criterion D alone $(n=222)$. Our final filtering step kept all assessments with reported estimates for the number of mature individuals $(n=171)$. One assessment was written without an English translation, and was removed from the dataset. The final, working dataset of 170 assessments included all species on the IUCN Red List with available population size estimates in the endangered (EN) category listed under Criterion D (small population size; note that IUCN uses the term "population size" but this refers to the entire species not individual populations). All filtering was done within Microsoft Excel.

We investigated the estimation and reporting of population sizes (i.e., estimates of mature individuals) in each assessment in the working dataset in order to quantify discrepancies between species' assessments. This included recording the number of assessments that provided primary sources (e.g., peer-reviewed publications) and methods of estimation (e.g., field surveys, camera traps, number of breeding pairs, etc.), as well as if the sources cited within the assessment (if applicable) provided methods of estimation. All information was retrieved from the text within the "Population" information segment of each assessment (available online at www.iucnredlist.org), as our working dataset contained species listed based on Criterion D alone (small population size) without consideration of habitat, threats, or other factors.

For species in the working dataset, we estimated $N_{\mathrm{e}}$ by multiplying the number of mature individuals reported in their assessments by 0.4 or 0.1 , representing a common range of $N_{\mathrm{e}} / N_{\mathrm{c}}$ ratios (Waples et al. 2011; Allendorf et al. 2013; Frankham et al. 2014). The maximum estimate was used for assessments that reported ranges of values for mature individuals. These species were then sorted into new IUCN Red List categories based on their estimated $N_{\mathrm{e}}$ in order to quantify the changes in risk categorization that would happen if the Red List considered $N_{\mathrm{e}}$. Under a genetics viewpoint, if the $N_{\mathrm{e}}$ estimate was less than 50 individuals, the species might be moved from its original EN category into the critically endangered (CR) category, per IUCN Red List Criterion D guidelines.

Thirty-nine assessments of 170 within our working dataset reported generation length. For these 39 species, we estimated loss of heterozygosity over the next 100 years using the following equation (Wright 1931):

$\%$ heterozygosity remaining $=\left[1-\left(1 /\left(2 * N_{\mathrm{e}}\right)\right]^{\mathrm{t}}\right.$

where $N_{\mathrm{e}}$ was estimated using the $N_{\mathrm{e}} / N_{\mathrm{c}}$ ratios of 0.4 and 0.1 , $N_{\mathrm{c}}$ is directly from the Red List assessment (reported as the number of mature adults), and $t$ is the number of generations in 100 years. The number of generations $(t)$ was estimated by dividing 100 by the generation length reported within the Red List assessments.

\section{Results}

Since 2001 and in the present guidelines, the number of "mature individuals" is defined as "the number of individuals known, estimated or inferred to be capable of reproduction"; therefore, population sizes for IUCN Red List listed species are contingent upon reproductive maturity (IUCN 2001; IUCN 2019). While the text in versions 6 through 14 (years 2006 to 2019) includes using lower population size estimations in cases of biased adult or breeding sex ratios, Version 13 (2017) added the following text explicitly regarding effective population size, which is still present in the current guidelines:

Note that effective population size $\left(N_{\mathrm{e}}\right)$ cannot be used as an estimate of the number of mature individuals. One reason is that reproductively suppressed individuals do not contribute to the calculation of $N_{\mathrm{e}}$, but, as explained above, they may be counted as mature individuals.

In our working dataset of 170 assessments, $96(\sim 56 \%)$ assessments did not report any primary, peer-reviewed literature within the "Population" section of text in relation to the estimate given for population size (i.e., the number of mature individuals). Sixty-three assessments of the 96 lacked any type of cited source for the estimation of mature individuals, and the other 33 cited secondary sources, personal communications, and unpublished results. Additionally, 131/170 ( $77 \%$ ) did not report the method of estimating the number 
of mature individuals. For the 39 assessments that provided a primary source citation for the mature individual estimates, 28 reported a methodology for the estimation. While 76/170 ( $45 \%)$ of the assessments reported a range of values for this estimate, uncertainty of the estimate was never discussed or estimated within the "Population" section text.

No assessments in the final dataset relayed the information necessary to estimate $N_{\mathrm{e}}$ based on demographic data (e.g., sex ratio, variance in family size or reproductive success, etc.), verified by manual inspection. When using an estimated $N_{\mathrm{e}} / N_{\mathrm{c}}$ ratio of $0.4,42$ species $(24.7 \%)$ had an estimated effective population size below 50 , and thus could warrant moving from the EN to CR threat category. When using an estimated $N_{\mathrm{e}} / N_{\mathrm{c}}$ ratio of $0.1,168(98.8 \%)$ EN species had an estimated effective size $<50$, and could likewise warrant moving into the CR category (Fig. 1; Online Resource 1).

When estimating loss of heterozygosity over the next 100 years, the average $H$-loss among species (estimated by 0.4 and $0.1 N_{\mathrm{e}} / N_{\mathrm{c}}$ ratios) was $9 \%$ for an $N_{\mathrm{e}} / N_{\mathrm{c}}$ ratio of 0.4 and $29 \%$ for an $N_{\mathrm{e}} / N_{\mathrm{c}}$ ratio of 0.1 (Fig. 2; Online Resource 2). Only 13 or 1 (using 0.4 and $0.1 N_{\mathrm{e}} / N_{\mathrm{c}}$ ratios) of these 39 species with reported generation times are expected to retain $>95 \%$ heterozygosity.

\section{Discussion}

Over the past decade, several calls have been made to apply conservation genetics to policy (Laikre 2010; Storfer et al. 2010; Hoban et al. 2013; Garner et al. 2016). In spite of several examples, there remains a frequent disconnect between genetic concepts and data and conservation policies and management (Santamaría and Mendez 2012). Here we used a range of common estimations to help bridge fundamental genetic concepts and one of the largest conservation instruments available, the IUCN Red List. Under current IUCN Red List guidelines, practitioners do not report effective population size $\left(N_{\mathrm{e}}\right)$ estimates, or metrics needed to estimate $N_{\mathrm{e}}$, to compliment the number of mature adults $\left(N_{\mathrm{c}}\right)$, despite the utility of $N_{\mathrm{e}}$ in risk assessment for populations. There is reason for this- if some species were assessed using both $N_{\mathrm{e}}$ and $N_{\mathrm{c}}$ while others were assessed only by $N_{\mathrm{c}}$, the Red List might be less useful for prioritizing or ranking conservation action, as species often need to be assessed with the same criteria. Nonetheless, the clear relevance of $N_{\mathrm{e}}$ for species and population survival cannot be ignored in the Red List or in conservation assessments broadly.

The Red List defines population size (again, note that for the IUCN Red List this refers to the species as a whole and not individual populations) as the number of mature individuals capable of reproduction; however, the number of individuals that successfully reproduce (and produce (a) $\mathrm{Nc}$

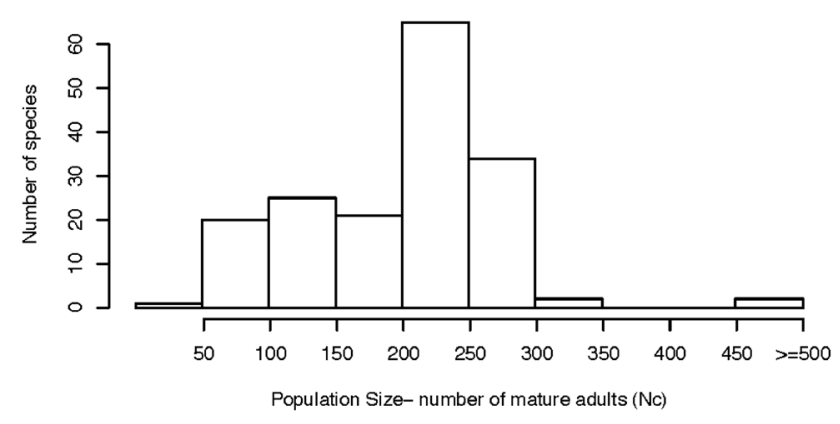

(b) Ne using $\mathrm{Ne}=0.4^{\star} \mathrm{Nc}$

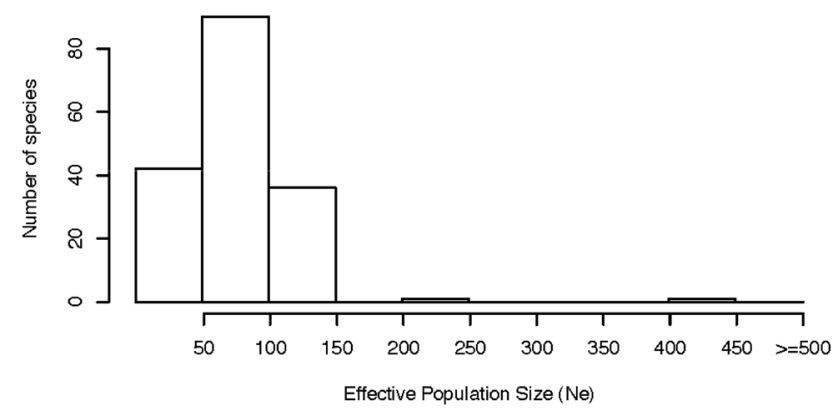

(c) $\mathrm{Ne}$ using $\mathrm{Ne}=0.1^{*} \mathrm{Nc}$

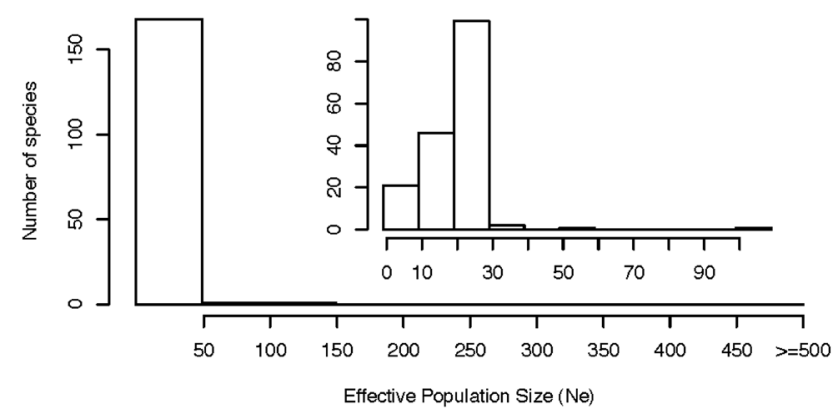

Fig. 1 Histograms of a population census size $N_{\mathrm{c}}$ (i.e., number of mature individuals reported within the Red List), b $0.4 N_{\mathrm{c}}$ (representing $\left.N_{\mathrm{e}} / N_{\mathrm{c}}=0.4\right)$, and c) $0.1 N_{\mathrm{c}}$ (representing $\left.N_{\mathrm{e}} / N_{\mathrm{c}}=0.1\right)$

offspring that survive to maturity) is generally much lower than the number that are capable of reproducing. Furthermore, in many cases there is a large variance in reproductive output, so the concept of effective population size is a highly useful complement to $N_{\mathrm{c}}$ when assessing population viability (Frankham 1995; Lacy 2019).

In our analyses, we found several issues in how IUCN assessments report on the number of mature individuals; addressing these issues would make it easier to more precisely estimate $N_{\mathrm{e}}$ and loss of heterozygosity. We strongly recommend reporting demographic metrics necessary for estimating $N_{\mathrm{e}}$ (e.g., number of mature or reproducing males and females, adult sex ratio, longevity, family size variance, 


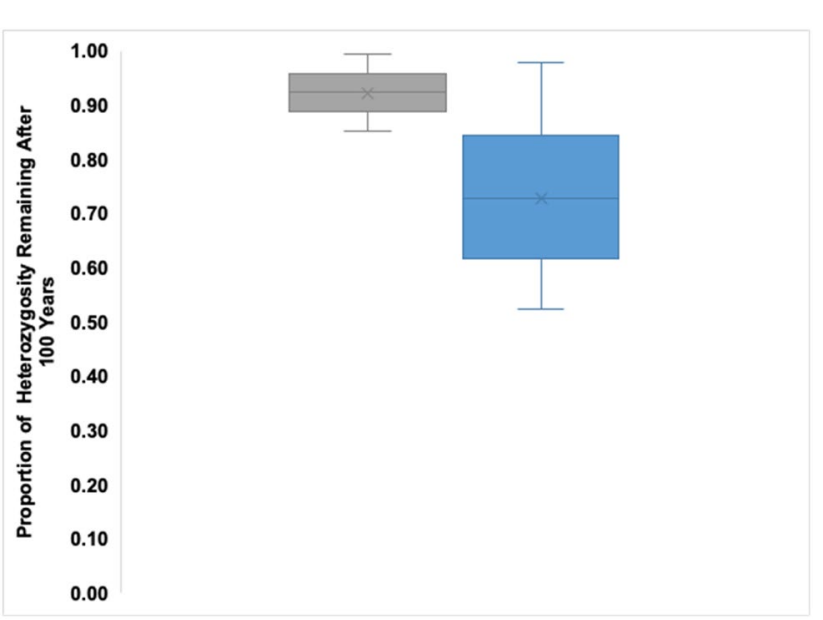

Fig. 2 Box plots showing the estimated remaining heterozygosity for 39 species after the next 100 years assuming an $N_{\mathrm{e}} / N_{\mathrm{c}}$ ratio of 0.4 (grey), and 0.1 (blue)

etc.). This will help practitioners to calculate $N_{\mathrm{e}}$ and also collaborate with geneticists (as recommended by Holderegger et al. 2019) to improve threat categorizations and reduce extinction risk and rates. Additionally, to improve standardization in reporting, we recommend assessors provide details of methods used and more explicitly highlight uncertainty in estimates of population size. It would be also valuable if information could be included, in a standardized way, on the history of a species' demographic decline (e.g., historic population size estimates and timing of decline), which could facilitate much more precise estimation of metrics including loss of heterozygosity (sensu Hoban et al. 2014) and number of generations at small $N_{\mathrm{e}}$. We recognize that both time and expense is a significant consideration when collecting data on the biological characteristics mentioned here. In situations where conditions are rapidly changing and a species is at a high risk of extinction, there might not be time to collect these data. Therefore, we do not argue that these measurements be taken no matter what, but rather that biologists should include them in assessments when possible as there is definitive value in recording such information.

The majority of species in our working dataset were plants, with $17 \%$ belonging to the Sorbus (rose family) genus. This bias likely reflects that there are many rare species in this genus, but may reflect disproportionate Red List contributions from certain botanists or countries. To investigate a potential taxonomic bias in the results, we removed these species from the working dataset and re-calculated the percent of species with an estimated effective population size below 50 (and thus potentially warranting a move from the EN to CR threat category), again using $N_{\mathrm{e}} / N_{\mathrm{c}}$ of 0.4 and 0.1 . However, the results we obtained when removing these species were similar to the original results, with $17.1 \%$ when using $N_{\mathrm{e}} / N_{\mathrm{c}}=0.4$ (compared to $24.7 \%$ before the removal of
Sorbus species) and $99.3 \%$ when using $N_{\mathrm{e}} / N_{\mathrm{c}}=0.1$ (compared to $98.8 \%$ before the removal of Sorbus species).

Additionally, we found that close to $1 / 4(51 / 222)$ of the assessments listed as EN under Criterion D (i.e., population size $<250$ ) didn't report a population size estimate, despite Criterion D being contingent upon this estimate. In cases like this where populations are small but estimating a census size is difficult, the use of genetic markers and an acceptance of $N_{\mathrm{e}}$ as a metric under Criterion D could provide more information that what is currently available. Sometimes it is easier to estimate $N_{\mathrm{e}}$ using genetic markers than to estimate $N_{\mathrm{c}}$ by traditional methods, because genetic markers can be applied to scat, hair, feathers, or other remnants of an individual (Taberlet et al. 1999). Genetic markers can be used to estimate $N_{\mathrm{c}}$ through capture-mark-recapture (CMR) methods, and additionally, genetic analysis costs are plummeting and transfer of markers between species is increasingly feasible (e.g., Andrews et al. 2016).

Effective population size estimates can vary widely depending on sampling, assumptions, the estimator, and more. While our "rule of thumb" estimates of $N_{\mathrm{e}} / N_{\mathrm{c}}$ equal to 0.4 and 0.1 represent a wide range and a simplistic approach, they are still useful in determining the range of likely actual $N_{\mathrm{e}}$ value. Using the larger 0.4 estimate still resulted in close to $1 / 4$ of the EN species having an estimated $N_{\mathrm{e}}<50$, meaning they could be candidates for the CR category. Other groups have suggested that an $N_{\mathrm{e}} / N_{\mathrm{c}}$ ratio of 0.14 is actually an overestimate for many species (Palstra and Ruzzante 2008), meaning our results using a ratio of 0.1 could often be closer to reality. In the 0.1 case, all but two of the 170 species would be moved into the CR category. Though it is theoretically possible that species sorted into threat categories based on small population sizes could have experienced genetic purging and are less susceptible to genetic effects, most threatened species are recently declining and have not been at low populations over long periods of time. While we recognize the potential pitfalls in using the same $N_{\mathrm{e}} / N_{\mathrm{c}}$ ratio across taxa, this study represents a critical starting point and highlights a key message-many species are in a critical situation due to small effective population size, inbreeding, loss of variation, fixation of deleterious alleles, and reduced long-term viability. The IUCN Red List status of "endangered" (EN) may not highlight the urgency of this situation, and we aim to make clear to non-genetics experts the scope of this problem.

One major asset of the Red List is its relatively unchanged nature over time, and hence the ability to track temporal changes. Nonetheless, our findings suggest many species may have effective population sizes substantially below recommended conservation thresholds $\left(N_{\mathrm{e}}<50\right)$ and that the Red List criteria overlook important genetic and evolutionary processes like strong genetic drift, inbreeding, and loss of heterozygosity. This and previous findings that genetic 
diversity is not well predicted by RL status argue that there is room for improvement in the incorporation of genetic considerations into the IUCN Red List. Willoughby et al. (2015) examined the relationship between IUCN RL category and microsatellite diversity and determined whether IUCN criteria are effective at identifying low genetic diversity species; generally, genetic diversity did not correlate with IUCN Red List category. The team suggested a genetic IUCN criterion with a conceptual outline that includes estimation of census size, effective population size, neutral genetic diversity, reference genetic diversity, and number of generations until reaching a certain heterozygosity-loss cutoff value. Here, we are also suggesting that $N_{\mathrm{e}}$ (and the predicted loss of heterozygosity) be considered as additional important information to supplement reporting and assessments because genetic factors (e.g., drift, inbreeding depression) reduce population persistence (Allendorf and Ryman 2002; Lacy 2019).

Feasible solutions may include a new category for listing, e.g., "Category F: Effective Population Size $<50$ ", or creation of an alternative (additional or independent) list including genetic factors in assessments (e.g., a "Red-Genes List"). Lists complementary to the Red List, e.g., the Green List, have proven successful (Akçakaya et al. 2018). Other assessment devices exist including the national endangered species lists, the NatureServe list, BGCI ThreatList, and the European Union Habitats Directive, and these could consider $N_{\mathrm{e}}$. Additionally, IUCN guidelines could be updated to include suggested, but optional, fields for reporting demographic and life history metrics (e.g., sex ratio, family size variation, mating strategy, birth and death rates, reproductive output) when possible, which can be used by conservation geneticists and other practitioners to estimate $N_{\mathrm{e}}$ separately and precisely for each species.

Our results also have usefulness in the context of the Convention on Biological Diversity Target 13 on "genetic erosion". The Red List Index is currently an indicator for this Target but we suggest that a rule of thumb such as $0.4 N_{\mathrm{c}}$ below thresholds such as 50 and 500 could be complementary with more genetic relevance and a better predictor of extinction risk (Laikre et al. 2020, Hoban et al. 2020). We acknowledge that this approach is imperfect, but including genetic factors (which have known influences on population persistence) is an improvement over no indicators at all. We hope these results and perspectives motivate improved risk assessment and conservation of threatened species while advancing quantitative biodiversity monitoring broadly.

Acknowledgements We thank the IUCN Red List of Threatened Species for providing data and ongoing listing efforts, and the W.A. Franke Endowed Graduate Fellowship in Wildlife Biology for funding and support of BAG. GL was supported in part by Grants from NSF-1639014 and National Aeronautics and Space Administration. S.H acknowledges funding from The Center for Tree Science, The Morton Arboretum.
Availability of data and material The datasets generated during and/or analyzed during the current study are available at https://github.com/ brgrnr/IUCN_Ne_data.

\section{Compliance with ethical standards}

Conflict of interest The authors declare that they have no conflict of interest.

Open Access This article is licensed under a Creative Commons Attribution 4.0 International License, which permits use, sharing, adaptation, distribution and reproduction in any medium or format, as long as you give appropriate credit to the original author(s) and the source, provide a link to the Creative Commons licence, and indicate if changes were made. The images or other third party material in this article are included in the article's Creative Commons licence, unless indicated otherwise in a credit line to the material. If material is not included in the article's Creative Commons licence and your intended use is not permitted by statutory regulation or exceeds the permitted use, you will need to obtain permission directly from the copyright holder. To view a copy of this licence, visit http://creativecommons.org/licenses/by/4.0/.

\section{References}

Akçakaya HR et al (2018) Quantifying species recovery and conservation success to develop an IUCN Green List of Species. Conserv Biol 32(5):1128-1138

Allendorf FW, Ryman N (2002) The role of genetics in population viability analysis. In: Beissinger SR, McCullough DR (eds) Population viability analysis. University of Chicago Press, Chicago, pp 50-85

Allendorf FW et al (2013) Conservation and the genetics of populations, 2nd edn. Wiley-Blackwell, Hoboken

Andrews KR et al (2016) Harnessing the power of RADseq for ecological and evolutionary genomics. Nat Rev Genet 17(2):81-92

Beaumont M, Wang J (2019) Conservation genetics. In: Balding J, Moltke I, Marioni J (eds) Handbook of statistical genomics, vol 1, 4th edn. Wiley, Hoboken

Beletsky LD, Orians GH (1989) A male red-winged blackbird breeds for 11 years. Northwestern Nat 70:10-12

Bozzuto C et al (2019) Inbreeding reduces long-term growth of Alpine ibex populations. Nat Ecol Evol 3:1359-1364

Crow JF, Kimura M (1970) Introduction to population genetics theory. Harper \& Row, London

Dunham J et al (1999) Assessing extinction risk: integrating genetic information. Conserv Ecol 3(1):2

Ficetola GF et al (2010) Polygyny, census and effective population size in the threatened frog, Rana latastei. Anim Conserv 13(S1):82-89

Fisher RA (1930) The genetical theory of natural selection. Oxford University Press, Oxford

Frankham R (1995) Effective population size/adult population size ratios in wildlife: a review. Genet Res 66:95-107

Frankham R et al (2002) Introduction to conservation genetics. Cambridge University Press, Cambridge

Frankham R et al (2014) Genetics in conservation management: revised recommendations for the 50/500 rules, Red List criteria and population viability analyses. Biol Conserv 170:56-63

Garner BA et al (2016) Genomics in conservation: case studies and bridging the gap between data and application. Trends Ecol Evol 31:81-83 
Greenbaum G et al (2017) Revealing life-history traits by contrasting genetic estimations with predictions of effective population size. Conserv Biol 32:817-827

Hauser L et al (2002) Loss of microsatellite diversity and low effective population size in an overexploited population of New Zealand snapper (Pagrus auratus). Proc Natl Acad Sci USA 99:11742-11747

Hoarau G et al (2005) Low effective population size and evidence for inbreeding in an overexploited flatfish, plaice (Pleuronectes platessa L.). Proc R Soc Lond B 272:497-503

Hoban SM et al (2013) Bringing genetic diversity to the forefront of conservation policy and management. Conserv Genet Resour 5:593-598

Hoban S et al (2014) Comparative evaluation of potential indicators and temporal sampling protocols for monitoring genetic erosion. Evol Appl 7:984-998

Hoban S et al (2020) Genetic diversity targets and indicators in the CBD post-2020 Global Biodiversity Framework must be improved. Biol Conserv 248:108654

Holderegger R et al (2019) Conservation genetics: linking science with practice. Mol Ecol 28:3848-3856

IUCN (2001) IUCN Red List Categories and Criteria: version 3.1. IUCN, Gland and Cambridge

IUCN Standards and Petitions Subcommittee (2019) Guidelines for using the IUCN Red List Categories and Criteria. Version 14. Prepared by the Standards and Petitions Subcommittee. http:// www.iucnredlist.org/documents/RedListGuidelines.pdf

Kendall KC et al (2016) Density, distribution, and genetic structure of grizzly bears in the Cabinet-Yaak Ecosystem. J Wildl Manag 80(2):314-331

Lacy RC (2019) Lessons from 30 years of population viability analysis of wildlife populations. Zoo Biol 38:67-77

Laikre L (2010) Genetic diversity is overlooked in international conservation policy implementation. Conserv Genet 11:349-354

Laikre L et al (2009) Importance of genetics in the interpretation of favourable conservation status. Conserv Biol 23:1378-1381

Laikre L et al (2020) Post-2020 goals overlook genetic diversity. Science 367:1083-1085

Mace GM, Lande R (1991) Assessing extinction threats: toward a reevaluation of IUCN threatened species categories. Conserv Biol $5: 148-157$

Newman D, Pilson D (1997) Increased probability of extinction due to decreased genetic effective population size: experimental populations of Clarkia pulchella. Evolution 51(2):354-362

Norse EA et al (1986) Conserving biological diversity in our national forests. Wilderness Society, Washington

Palstra FP, Ruzzante DE (2008) Genetic estimates of contemporary effective population size: what can they tell us about the importance of genetic stochasticity for wild population persistence? Mol Ecol 17:3428-3447

Pierson JC et al (2016) Genetic factors in threatened species recovery plans on three continents. Front Ecol Environ 14(8):433-440
Reed DH, Frankham R (2003) Correlation between fitness and genetic diversity. Conserv Biol 17:230-237

Santamaría L, Mendez PF (2012) Evolution in biodiversity policy-current gaps and future needs. Evol Appl 5:202-218

Spigler RB et al (2017) Inbreeding depression and drift load in small populations at demographic disequilibrium. Evolution 71:81-94

Storfer A et al (2010) Landscape genetics: where are we now? Mol Ecol 19:3496-3514

Sun X, Hedgecock D (2017) Temporal genetic change in North American Pacific oyster populations suggests caution in seascape genetics analyses of high gene-flow species. Mar Ecol Prog Ser 565:79-93

Taberlet $P$ et al (1999) Noninvasive genetic sampling: look before you leap. Trends Ecol Evol 14:323-327

Ujvari B et al (2018) Genetic diversity, inbreeding and cancer. Proc R Soc B 285:20172589

Wang J (2016) Pedigrees or markers: which are better in estimating relatedness and inbreeding coefficient? Theor Popul Biol 107:4-13

Wang J et al (2016) Prediction and estimation of effective population size. Heredity 117:193-206

Waples RS (2005) Genetic estimates of contemporary effective population size: to what time periods do the estimates apply? Mol Ecol $14: 3335-3352$

Waples RS (2016) Tiny estimates of the Ne/ $\mathrm{N}$ ratio in marine fishes: are they real? J Fish Biol 89:2479-2504

Waples RS, Do C (2008) LdNe: a program for estimating effective population size from data on linkage disequilibrium. Mol Ecol Resour 8:753-756

Waples RS et al (2011) Calculating $\mathrm{Ne}$ and $\mathrm{Ne} / \mathrm{N}$ in age-structured populations: a hybrid Felsenstein-Hill approach. Ecology 92:1513-1522

Waples RS et al (2013) Simple life history traits explain key effective population size ratios across diverse taxa. Proc Biol Sci 280:20131339

Waples RS et al (2014) Effects of overlapping generations on linkage disequilibrium estimates of effective population size. Genetics 197:769-780

Willoughby JR et al (2015) The reduction of genetic diversity in threatened vertebrates and new recommendations regarding IUCN conservation rankings. Biol Conserv 191:495-503

Wright S (1931) Evolution in Mendelian populations. Genetics 16:97-159

Publisher's Note Springer Nature remains neutral with regard to jurisdictional claims in published maps and institutional affiliations. 